\title{
Performance Comparison Between Carbon Nanotube and Copper Interconnects for Gigascale Integration (GSI)
}

\author{
Azad Naeemi, Senior Member, IEEE, Reza Sarvari, Student Member, IEEE, and James D. Meindl, Life Fellow, IEEE
}

\begin{abstract}
Physical models are used to determine the ultimate potential performance of carbon nanotube interconnects and compare them with minimum-size copper wires implemented at various technology generations. Results offer important guidance regarding the nature of carbon nanotube technology development needed for improving interconnect performance. Since wave propagation is slow in a single nanotube, nanotube bundles with larger wave speeds must be used. At the $45-\mathrm{nm}$ node (year 2010), the performance enhancement that can be achieved by using nanotube bundles is negligible, and at the $22-\mathrm{nm}$ node (year 2016) it can be as large as $80 \%$.
\end{abstract}

Index Terms-Interconnections, kinetic inductance, modeling, molecular electronics, quantum wires.

\section{INTRODUCTION}

A $S$ interconnect feature sizes shrink, copper resistivity increases due to surface and grain boundary scatterings and also surface roughness [1]. Furthermore, wires, especially power and ground lines, are becoming more and more vulnerable to electromigration because of rapid increases in current densities [2]. In contrast, carbon nanotubes exhibit a ballistic flow of electrons with electron mean-free paths of several micrometers, and are capable of conducting very large current densities [3]. Carbon nanotubes are therefore proposed as potential candidates for signal and power interconnection [4], [5]. In this letter, after reviewing the circuit models for single-wall carbon nanotubes (SWCN), the performances of copper wires and nanotube interconnects are compared. The results offer important guidance regarding the nature of carbon nanotube technology development needed for improving interconnect performance.

\section{Circuit Models for Metallic Carbon Nanotubes}

Neglecting electron spin and sublattice degeneracy, the dc conductance of an ideal (ballistic) quantum wire is independent of length and is equal to $e^{2} / h$, where $h$ is the Plank constant, and $e$ is electron charge [6]. The current carriers of a nanotube occupy the one-dimensional conduction bands with very low density of states, and hence the kinetic energy stored in current is

Manuscript received October 15, 2004; revised November 19, 2004. The review of this letter was arranged by Editor S. Kawamura.

The authors are with the Georgia Institute of Technology, Atlanta, GA 30332 USA (e-mail: azad@ece.gatech.edu).

Digital Object Identifier 10.1109/LED.2004.841440 so large that it results in a very large kinetic inductance per unit length [6]-[8]

$$
l_{k}=\frac{h}{\left(2 e^{2} v_{F}\right)}
$$

where $v_{F}$ is the Fermi velocity [8]. For graphene and hence carbon nanotubes $v_{F}=8 \times 10^{5} \mathrm{~m} / \mathrm{s} \mathrm{[6],} \mathrm{[7].} \mathrm{The} \mathrm{kinetic} \mathrm{induc-}$ tance per unit length of carbon nanotubes is therefore around $16 \mathrm{nH} / \mu \mathrm{m}$, more than four orders of magnitude larger than its magnetic counterpart.

To add electric charge to a quantum wire, one must add electrons to available states above the Fermi level (Pauli exclusion principle); hence, there is a quantum capacitance of

$$
c_{Q}=\frac{2 e^{2}}{\left(h v_{F}\right)}
$$

in series with the electrostatic capacitance [6]-[8]. The quantum capacitance of a carbon nanotube is $100 \mathrm{aF} / \mu \mathrm{m}$, and is of the same order of magnitude as its electrostatic counterpart.

Electron spin and sublattice degeneracy result in four parallel channels through which three spinmodes and one chargemode can travel [6]. The wave propagation speed for the charge mode is

$$
v=\sqrt{\frac{4}{l_{K}}\left(\frac{1}{c_{E}}+\frac{1}{4 c_{Q}}\right)} .
$$

Assuming that the electrostatic and quantum capacitances are equal, the wave speed is $\sqrt{5} v_{F}=1.78 \times 10^{6} \mathrm{~m} / \mathrm{s}$, which is 162 times smaller than the speed of light in free space. The speed of spin-modes through which no charge is transferred is always $v_{F}$ regardless of nanotube electrostatic capacitance [6].

\section{IDEAL CARbon Nanotubes Versus Copper Wires}

For the 22-nm node (year 2016), latencies of ideal carbon nanotube and copper interconnects are plotted versus length in Fig. 1. For copper interconnects, the distributed $R C$ model is used [9], and copper resistivity has been calculated by the combination of Fuchs-Sondheimer and Mayadas-Shatzkes models for surface scattering and grain boundary scattering [1], [10], respectively.

Due to large values of contact resistance and characteristic impedance of a single nanotube, many of them need to be used in parallel for interconnect applications. Compared to a single nanotube, a monolayer of nanotubes in parallel above a ground plane as shown in Fig. 1 has a smaller inductance. However, it has a larger capacitance. Hence, by connecting more and more 


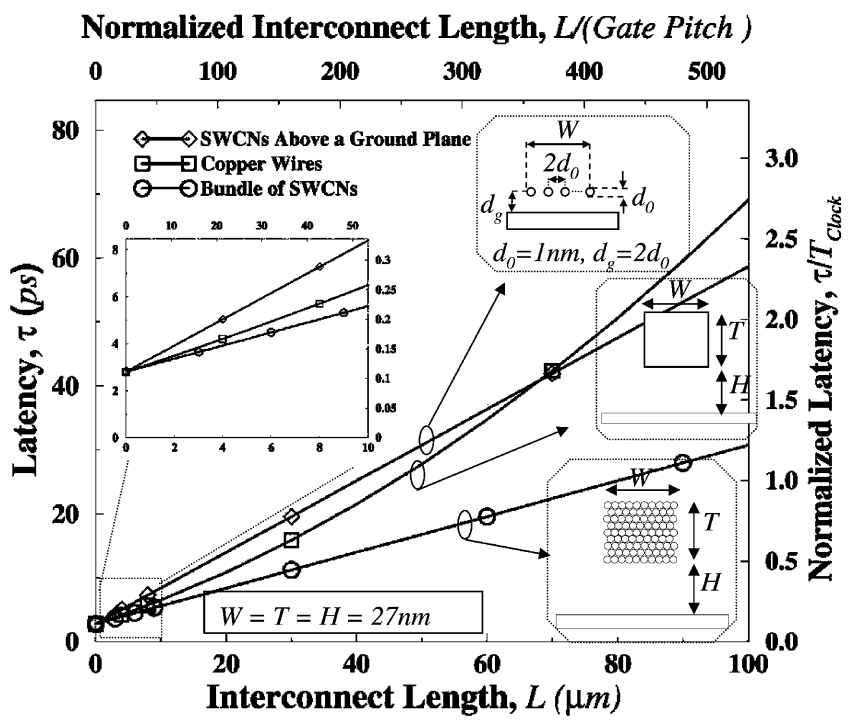

Fig. 1. Latency versus interconnect length for ideal single-layered carbon nanotubes above a ground plane, minimum-sized copper wires implemented at the 22-nm technology node (year 2016) and bundles of ideal SWCNs for $n>100$. For copper interconnects, surface scattering co-efficient, $p$, and grain boundary reflection coefficient, $R$, are pessimistically assumed to be 0 and 0.5 , respectively. Mean-free path of electrons in ideal carbon nanotubes is larger than their length.

nanotubes in parallel, the wave propagation speed does not improve significantly. Latency of such a "single layer" nanotube interconnect is therefore

$$
\tau_{\text {nano }}=0.7 R_{\text {tr }} C_{L}+\frac{L}{\left(\sqrt{5} v_{F}\right)}
$$

where $R_{\mathrm{tr}}$ is driver resistance (equal to the line characteristic impedance for impedance matching), $C_{L}$ is load capacitance, and $L$ is interconnect length.

In contrast, a bundle of closely packed parallel carbon nanotubes above a ground plane as shown in Fig. 1 has a capacitance per unit length approximately equal to that of a single nanotube. If all cross-sectional dimensions scale proportionally with increasing the number of nanotubes within the bundle, $n$, the overall capacitance per unit length remains constant (independent of $n$ ). The kinetic inductance per unit length, however, decreases linearly with $n$, because of which the wave propagation speed increases by $\sqrt{n}$ as (3) shows. At the contacts, good connections to all nanotubes within the bundle are needed such that all nanotubes can contribute to conduction. The distance between nanotubes within the bundle is desired to be as small as possible to have the largest nanotube density. It has been optimistically assumed that there is no unwanted quantum coupling between nanotubes within the bundle. Further investigation is needed to verify this assumption even though preliminary measurements and analyzes indicate that the coupling between nanotubes within a bundle is weak [11], [12].

For $n>100$, the wave travel time becomes much smaller than the $R C$ charge up time of most typical interconnects even at the end of the International Technology Roadmap for Semiconductors, hence, latency would be equal to

$$
\tau_{\text {bundle }}=0.7 R_{\text {tr }}\left(c_{\text {bundle }} L+C_{L}\right)
$$

where $c_{\text {bundle }}$ is capacitance per unit length of the bundle of nanotubes. Since the diameter of SWCNs can be less than $1 \mathrm{~nm}$
[13], a bundle of, for instance, $400 \mathrm{SWCNs}$ can be as narrow as $20 \mathrm{~nm}$.

\section{Nonideal CARbon NANOtubes Versus COPPER WIRES}

Even initially ideal carbon nanotubes become disordered once they are physisorbed on a surface [3]. Electron mean-free path, $L_{0}$, in real carbon nanotubes is, hence, finite and their resistance increases with length. There are inconsistent results published in literature, both experimental and theoretical, regarding the dependency of resistance on length; some indicating an exponential relationship $\left(R=R_{0} \exp \left(L / 2 L_{0}\right)\right)$ [14], [15], and some showing a linear dependency $\left(R=R_{0}\left(1+L / L_{0}\right)\right)$ [16], [17], where $R_{0}$ is the resistance of a bundle of ballistic nanotubes (ideally equal to $h /\left(4 e^{2} n\right)$ ). In this section, these two possible dependencies are considered separately.

$$
\text { Exponential Dependency }\left(R=R_{0} e^{\frac{L}{2 L_{0}}}\right) \text {. }
$$

In the inset in Fig. 2, latencies of SWNT bundles and copper interconnects implemented at the 22-nm node are plotted versus interconnect length for $L_{0}=5 \mu \mathrm{m}$ and $L_{0}=10 \mu \mathrm{m}$. There is a length beyond which latency of SWNT bundles becomes larger than that of copper wires. As a rule of thumb, this critical length is roughly ten times the electron mean-free path in SWCNs. The performance enhancement that can be achieved by using nanotube bundles is a function of interconnect length, and its maximum is

$$
\begin{aligned}
& \left.\frac{\tau_{\text {copper }}-\tau_{\text {Bundle }}}{\tau_{\text {Bundle }}}\right|_{\max } \\
& \approx \max \left\{\left[0.76 \frac{L_{0} r_{\text {int }}}{R_{\text {tr }}}\left(\ln \frac{2 R_{\text {tr }}}{R_{0}}-1\right)-0.24\right], 0\right\}
\end{aligned}
$$

which is plotted in Fig. 2 versus electron mean-free path for four various technology generations. It can be seen that the performance enhancement at the 45-nm node (year 2010) is negligible even if mean-free paths as large as 10 or $20 \mu \mathrm{m}$ are achieved. This is mainly due to small resistance per unit length of copper wires. At the 22-nm node (year 2016), however, nanotube bundles are $30 \%$ faster for mean-free path of $5 \mu \mathrm{m}$, and they can be $80 \%$ faster if achieving a mean-free path of $10 \mu \mathrm{m}$ is feasible. Theoretical calculations show that electron mean-free path in SWCNs can be as large $10 \mu \mathrm{m}$ [3].

$$
\text { Linear Dependency }\left(R=R_{0}\left(1+\frac{L}{L_{0}}\right)\right) \text {. }
$$

In the inset in Fig. 3, latencies of SWNT-bundles and copper interconnects implemented at the 22-nm node are plotted versus interconnect length for $L_{0}=0.1 \mu \mathrm{m}, 1 \mu \mathrm{m}$ and $10 \mu \mathrm{m}$ assuming that nanotube resistance increases linearly with length. It can be seen that nanotube bundles outperform copper wires unless they have very short mean-free paths $(\sim 0.1 \mu \mathrm{m})$. The speed improvement that can be attained by using nanotube bundles is

$$
\frac{\tau_{\text {copper }}-\tau_{\text {Bundle }}}{\tau_{\text {Bundle }}} \approx \frac{R_{\mathrm{tr}}+0.5 r_{\text {int }} L}{R_{\mathrm{tr}}+\frac{0.5 R_{0} L}{L_{0}}}-1
$$

and it increases as interconnect length increases. This performance gain is plotted versus mean-free path in Fig. 3 for $100-\mu \mathrm{m}$-long interconnects implemented at four various technology generations. Again, it can be seen that the performance gain at the $45-\mathrm{nm}$ node is negligible whereas at $22-\mathrm{nm}$ node it 


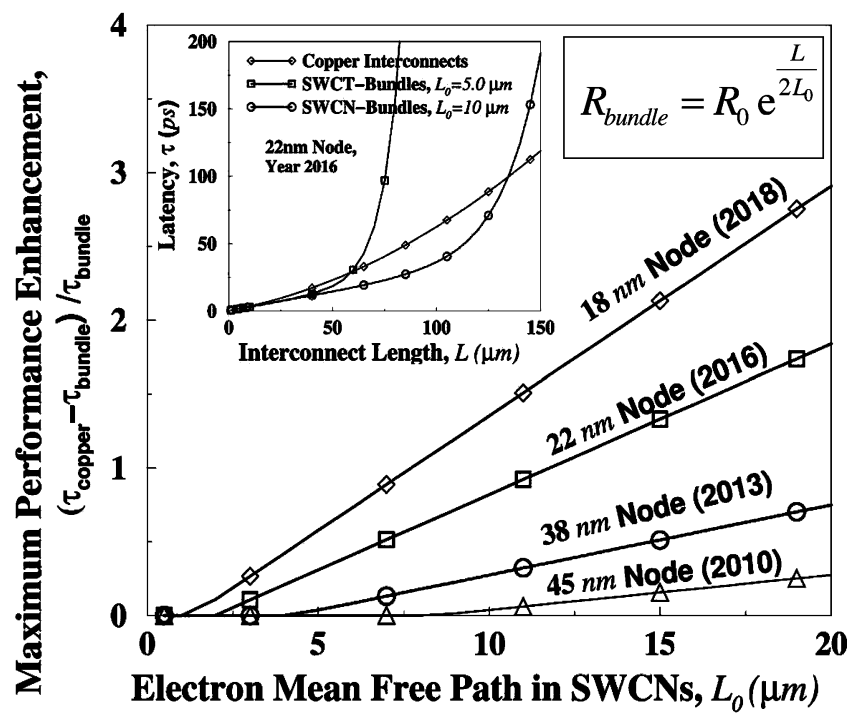

Fig. 2. Inset: Latency versus interconnect length for minimum-sized copper wires implemented at the $22-\mathrm{nm}$ node and bundles of SWCNs with electron mean-free paths of 5 and $10 \mu \mathrm{m}$ assuming that SWCN resistance increases exponentially with length. For copper interconnects $p=0$ and $R=0.5$ are assumed. Main plot: Maximum performance enhancement that can be achieved by using carbon nanotubes versus electron mean-free path in nanotubes for four various generations of technology. At the $22-\mathrm{nm}$ node (year 2016) nanotube bundles can be $30 \%$ faster than copper wires for mean-free path of $5 \mu \mathrm{m}$, and they can be $80 \%$ faster if achieving a mean-free path of $10 \mu \mathrm{m}$ is feasible.

can be as large as $80 \%$ if mean-free paths of around $1 \mu \mathrm{m}$ are available. It should be noted that mean-free paths longer than $1 \mu \mathrm{m}$ have already been reported [16], [17].

For large voltage biases $(>160 \mathrm{mV})$, electrons can be backscattered by optical or zone-boundary phonons [18]. Resistance of carbon nanotube interconnects, therefore, increases if the voltage drop along them is large. The relative increase in resistance of nanotubes is a function of the electric field along them. A rigorous analysis that will be published elsewhere shows that for interconnect applications, the electric field inside nanotubes is small enough and the low-bias analysis is quite valid.

\section{CONCLUSION}

Using physical models, the performances of SWCN and minimum-size copper interconnects are compared for various technology generations. Due to the low density of states in all quantum wires including carbon nanotubes, SWCNs have an extremely large kinetic inductance which causes slow wave propagation. It is therefore critical to use bundles of densely packed nanotubes to improve the wave speed. Carbon nanotubes fabricated so far have shown either linear or exponential resistance increases with length. Analyzing both cases has shown that the performance advantage of nanotube bundles for the 45 -nm node (year 2010) is negligible. At the 22-nm node (year 2016), however, nanotube bundles can be $80 \%$ faster than copper wires if electron mean-free paths in SWCNs are large enough ( 1 and $10 \mu \mathrm{m}$ for linear and exponential dependencies, respectively). Certainly, such a performance enhancement is contingent on many technological breakthroughs including fabrication of bundles of tens-of-micrometers long densely packed nanotubes with small contact resistances.

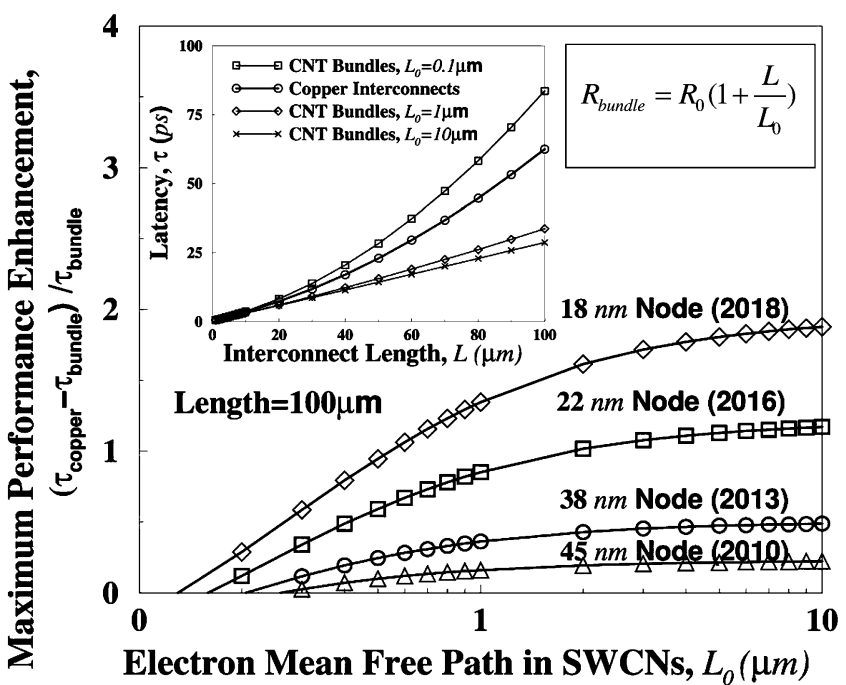

Fig. 3. Inset: Latency versus interconnect length for minimum-sized copper wires implemented at the 22-nm node and bundles of SWCNs with electron mean-free paths of $0.1,1$, and $10 \mu \mathrm{m}$ assuming that SWCN resistance increases linearly with length. For copper interconnects, $p=0$ and $R=0.5$ are assumed. Main plot: Maximum performance enhancement that can be achieved by using carbon nanotubes versus electron mean-free path in nanotubes for four various generations of technology. At the 22-nm node (year 2016) nanotube bundles can be $80 \%$ faster than copper wires if they have a mean-free path of $1 \mu \mathrm{m}$.

\section{REFERENCES}

[1] S. M. Rossnagel and T. S. Kaun, "Alteration of $\mathrm{Cu}$ conductivity in the size effect regime," J. Vac. Sci. Technol. B, Microelectron. Process. Phenom., vol. 22, no. 1, pp. 240-247, Jan./Feb. 2004.

[2] International Technology Roadmap for Semiconductors (ITRS), San Jose, CA, 2003

[3] C. T. White and T. N. Todorov, "Nanotubes go ballistic," Nature, vol. 411, pp. 649-651, Jun. 2001.

[4] V. Zhirnov, D. Herr, and M. Meyyappan, "Electronic applications of carbon nanotubes become closer to reality," J. Nanopart. Res., vol. 1, p. $151,1999$.

[5] J. Li et al., "Bottom-up approach for carbon nanotubes interconnects," Appl. Phys. Lett., vol. 82, pp. 2491-2493, Apr. 2003.

[6] P. J. Burke, "Lüttinger liquid theory as a model of the gigahertz electrical properties of carbon nanotubes," IEEE Trans. Nanotech., vol. 1, no. 5, pp. 129-144, Sep. 2002.

[7] R. Tarkinainen et al., "Multiwalled carbon nanotubes: Luttinger versus Fermi liquid," Phys. Rev. B, Condens. Matter, vol. 64, pp. 95412/1-95412/4, Oct. 2001.

[8] M. W. Bockrath, "Carbon nanotubes: Electrons in one dimension," Ph.D. dissertation, Univ. California, Berkeley, CA, 1999.

[9] H. B. Bakoglu, Circuit, Interconnections, and Packaging for VLSI. Reading, MA: Addison-Wesley, 1990.

[10] W. Steinhögl et al., "Size dependent resistivity of metallic wires in the mesoscopic range," Phys. Rev. B, Condens. Matter, vol. 66, pp. 075414/1-075414/4, Aug. 2002.

[11] H. Stahl, J. Appenzeller, R. Martel, P. Avouris, and B. Lengeler, "Intertube coupling in ropes of single-wall carbon nanotubes," Phys. Rev. Lett., vol. 85, pp. 5186-5189, 2000.

[12] M. Bockrath et al., Science, vol. 275, pp. 1922-1925, Mar. 1997.

[13] A. Bachtold et al., "Suppression of tunneling into mutliwall carbon nanotubes," Phys. Rev. Lett., vol. 87, pp. 166801/1-166801/4, Oct. 2001.

[14] P. J. de Pablo et al., "Nonlinear resistance versus length in single-walled carbon nanotubes," Phys. Rev. Lett., vol. 88, pp. 036804/1-036804/4, Jan. 2002.

[15] A. Andriotis, M. Menon, and L. Chernozatonski, "Non-linear resistance dependence on length in single-wall carbon nanotubes," Nano Lett., vol. 3, pp. 131-134, 2003.

[16] J. Y. Park, S. Rosenbelt, Y. Yaish, V. Sazonova, H. Üstunel, S. Braig, T. A. Arias, and P. L. McEuen, "Electron-phonon scattering in metallic single-walled carbon nanotubes," Nano Lett., vol. 4, pp. 517-520, 2004.

[17] S. Li, Z. Yu, C. Rutherglen, and P. J. Burke, "Electrical properties of $0.4 \mathrm{~cm}$ long single-walled carbon nanotubes," Nano Lett., vol. 4, pp. 2003-2007, 2004

[18] Z. Yao, C. L. Kane, and C. Dekker, "High-field electrical transport in single-wall carbon nanotubes," Phys. Rev. Lett., vol. 84, pp. 2941-2944, 2000. 